\title{
Hypoalgesic responses resulting from paired or separate exposures to naloxone and a heat stressor: Evidence from the tail-flick radiant heat test
}

\author{
H. FOO \\ Northern Territory University, Darwin, Northern Territory, Australia
}

\begin{abstract}
Rats given unpaired exposures to naloxone and the heated floor of a hot-plate apparatus acquired hypoalgesia, as evidenced by long latencies to paw-lick and tail-flick. Acquisition of hypoalgesia was found to be potentiated when preexposure to the heat stressor was paired with naloxone. The hypoalgesic responses detected with the tail-flick radiant heat test were reversed by naloxone and were not acquired by morphine-experienced rats, suggesting mediation by endogenous opioid mechanisms. The hypoalgesic responses can be modulated by cholecystokinin because the long tail-flick latencies were abolished by CCK-8. However, there was no evidence for a potentiation of these hypoalgesic responses by proglumide. There was also no evidence that naloxone had interacted with the heat stimulus of the tail-flick test employed to induce hypoalgesia. The present results are discussed in terms of their implications for using the tail-flick and hot-plate tests in studies investigating stress-induced hypoalgesia and morphine hypoalgesic tolerance.
\end{abstract}

Endogenous pain inhibitory mechanisms can be activated by Pavlovian conditioned stimuli (CSs) that have been paired with nociception (see Greeley \& Westbrook, 1990; Rochford \& Stewart, 1987b; Ross \& Randich, 1985; Westbrook, Greeley, Nabke, Swinbourne, \& Harvey, 1991). For example, rats tested for latencies to paw-lick (PL) where they have been exposed to a heated $\left(52^{\circ} \mathrm{C}\right.$ or $54^{\circ} \mathrm{C}$ ) floor of the hot-plate apparatus are hypoalgesic relative to those preexposed to a nonheated $\left(23^{\circ} \mathrm{C}\right)$ floor of the same apparatus (e.g., Cox \& Westbrook, 1994; Foo \& Westbrook, 1994; Good \& Westbrook, 1995; Greeley \& Westbrook, 1990; Harris \& Westbrook, 1994; Westbrook et al., 1991). The hypoalgesic responses so induced are mediated by conditioning processes, because the long PL latencies are extinguished when exposure to the heat stressor and to the subsequent pain test are interpolated with exposures to the nonheated floor (Cox \& Westbrook, 1994; Foo \& Westbrook, 1994; Greeley \& Westbrook, 1990; Harris \& Westbrook, 1994; Westbrook et al., 1991).

There is evidence that acquisition of the long latencies to $\mathrm{PL}$ can be potentiated by pairing exposure to the heat stressor with the opioid antagonist naloxone. Specifically, rats that have been exposed to the heat stressor with naloxone come to acquire even longer latencies to PL than do those that had received separate exposures to the drug

This research was supported by a grant from the Northern Territory University. The procedures described in this paper were approved by the NTU Animal Experimentation Ethics Committee. Naloxone- $\mathrm{HCl}$ and CCK-8 were generous gifts from E. I. duPont de Nemours \& Co., USA, and Bristol-Myers Squibb Co., USA, respectively. Correspondence should be addressed to H. Foo, Department of Psychology, Northern Territory University, Darwin, NT 0909, Australia (e-mail: h_foo@banks.ntu.edu.au). and the heated floor (Foo \& Westbrook, 1993; see also Foo, 1992, Foo \& Westbrook, 1991a, 1991b, 1991c, 1994, Poulos, Knoke, Le, \& Cappell, 1990, Rochford \& Stewart, 1987a, Westbrook \& Greeley, 1990, and Westbrook et a1., 1991). The ability of naloxone to potentiate acquisition of hypoalgesia is also evidenced by the increases in PL latencies observed in rats given drug-stressor pairings relative to the decreases in PL latencies among rats given saline-heat stressor ones (Bianchi \& Panerai, 1993; Greeley, Le, Poulos, \& Cappell, 1988; Kavaliers \& Innes, 1993; M. K. Walker, Le, Poulos, \& Cappell, 1991). These findings have been interpreted to mean that naloxone interacts with the heat stressor to produce hypoalgesia by selectively increasing the level of motivational arousal or facilitating the retention of aversive conditioning (see Gallagher, 1985; Greeley \& Westbrook, 1990; Izquierdo, 1979; Westbrook et al., 1991).

Three main findings suggest that the hypoalgesic responses induced by naloxone-heat stressor pairings are mediated by associative processes. First, the long PL latencies are contextually controlled, detected in the place that had been paired with exposure to the heated floor but not in one that had been paired with exposure to the nonheated floor (Westbrook \& Greeley, 1990). Second, the long latencies are observed in the absence of the drug when rats given naloxone-heat stressor pairings are tested on the heated floor with saline (e.g., Foo \& Westbrook, 1991a; Rochford \& Stewart, 1987a; Westbrook \& Greeley, 1990). Third, such latencies are extinguished by repeated exposures to the nonheated floor (Foo \& Westbrook, 1991a; Westbrook \& Greeley, 1990).

The hypoalgesic effects observed are not specific to reductions in nociceptive responding to acute pain induced by the heated floor of the hot-plate assay, because 
these effects are detected in rats tested for nociceptive sensitivity to formalin in the place where they had previously received separate or combined exposures to naloxone and the heat stressor (Foo \& Westbrook 1992, 1993, 1994; see also Good \& Westbrook, 1995, and Harris \& Westbrook, 1994). Although detected with both the hot-plate assay and the formalin assay, the conditioned hypoalgesic responses appear to be mediated by different mechanisms. On the one hand, the long PL latencies observed in rats conditioned and tested on the heated floor (i.e., hot-plate paradigm) were not reduced by naloxone and were unaffected by a history of exposures to morphine, thus suggesting mediation by nonopioid mechanisms (Foo, 1992; Westbrook \& Greeley, 1990; see also Rochford \& Stewart, 1987a, 1987b). On the other hand, the decreases in paw attendant behaviors observed in rats conditioned with the heated floor and subsequently tested with formalin (i.e., formalin paradigm) were reversed by naloxone and were prevented by a history of exposures to morphine, thereby suggesting mediation by opioid mechanisms (Foo \& Westbrook, 1992, 1993, 1994; Good \& Westbrook, 1995; Harris \& Westbrook, 1994). More specifically, the involvement of opioid mechanisms was not apparent immediately (1-2 $\mathrm{min}$ ) upon reexposure to the hot-plate apparatus cues but became evident at a later (3-5 min) stage of exposure to such cues (Good \& Westbrook, 1995; Harris \& Westbrook, 1994). When combined, these findings suggest that the test employed for nociceptive assessment can critically determine the nature of the conditioned hypoalgesic responses (see Litchman \& Fanselow, 1990).

The present series of experiments used the tail-flick (TF) radiant heat test to further examine the importance of the pain assay employed in determining the form of conditioned hypoalgesic responses resulting from preexposure to a heat stressor. Specifically, this study had four main aims. The first of these (Experiment 1) was to further generalize the hypoalgesic effects of separate or combined exposures to naloxone and a heated floor to rats tested for nociceptive responding with the TF radiant heat test (D'Amour \& Smith, 1941), a test that is different from either the hot-plate or the formalin test in that it evokes a response that is mediated primarily by spinal mechanisms (Grossman, Basbaum, \& Fields, 1982; Irwin, Houde, Bennett, Hendershot, \& Seevers, 1951). The second aim was to determine whether the hypoalgesic responses observed with the TF test are mediated by nonopioid mechanisms, like the hypoalgesic responses detected with the hot-plate test, or by opioid ones, like those detected with the formalin test (Foo, 1992; Foo \& Westbrook, 1994), using the criteria of reversibility by naloxone (Experiment 2) and cross-tolerance with morphine (Experiment 3). The third aim was to investigate a role for cholecystokinin (CCK) in mediating expression of the opioid hypoalgesic responses revealed by the TF test, by evaluating the effects of the agonist CCK-8 and the antagonist proglumide on the long latencies to TF. The final aim of this study was to examine whether naloxone interacts with the heat stimulus used in the TF test to induce hypoalgesic responses.

\section{EXPERIMENT 1}

Experiment 1 involved four groups that differed in terms of their history of exposure to the heated floor of the hot-plate apparatus across 2 consecutive conditioning days. Rats in Group Sal-Amb/Sal-Amb were never exposed to the heat stressor, receiving separate exposures to naloxone and the nonheated floor on each of the 2 conditioning days. Rats in Group Sal-Amb/Sal-Hot were exposed to the heat stressor on one occasion, receiving separate exposures to naloxone and the nonheated floor on the 1 st conditioning day, followed by separate exposures to naloxone and the heated floor on the 2nd conditioning day. Rats in Group Sal-Hot/Sal-Hot and Group Nal-Hot/Nal-Hot were exposed to the heat stressor twice, receiving respectively separate or combined exposures to naloxone and the heat stressor on each of the 2 conditioning days. This experiment was designed to show (1) that rats given separate exposures to a heated floor and to naloxone come to acquire hypoalgesic responses when tested for latencies to PL (Group Nal-Hot/NalHot + Group Sal-Hot/Sal-Hot vs. Group Sal-Amb/SalHot) or TF (Group Nal-Hot/Nal-Hot + Group Sal-Hot/ Sal-Hot + Group Sal-Amb/Sal-Hot vs. Group Sal-Amb/ $\mathrm{Sal}-\mathrm{Amb}$ ) and (2) that acquisition of hypoalgesia can be enhanced by pairing exposure to the heat stressor with the drug, as evidenced by latencies to PL (Group Nal-Hot/ Nal-Hot vs. Group Sal-Hot/Sal-Hot) or TF (Group NalHot/Nal-Hot vs. Group Sal-Hot/Sal-Hot + Group Sal$\mathrm{Amb} / \mathrm{Sal}$-Hot). Experiment 1 employed a within-subject design to demonstrate the hypoalgesic effects across both the hot-plate test and the TF test, rather than a betweensubject design, in order to conserve the number of animals used.

\begin{abstract}
Method
Subjects

The subjects were 20 experimentally naive, male Wistar rats, with an average weight of $257 \mathrm{~g}$ (range $220-301 \mathrm{~g}$ ). They were obtained from the colony maintained by the Laboratory Animal Services, University of Adelaide. The rats were housed in plastic boxes $(60 \times 40 \times 20 \mathrm{~cm})$ throughout the course of the experiment and were given free access to food and water. There were 5 rats to each box. The boxes were kept in a colony room maintained on a normal 12:12-h light:dark cycle, and the experiment was conducted during the light cycle.
\end{abstract}

\footnotetext{
Apparatus

The hot-plate apparatus consisted of a Plexiglas cylinder $(23 \mathrm{~cm}$ inner diameter $\times 48 \mathrm{~cm}$ high) with a brass floor ( $1 \mathrm{~mm}$ thick) that was fixed $12 \mathrm{~cm}$ above the base of the cylinder. The portion of the cylinder below the brass floor was perforated with holes $(2 \mathrm{~cm} \mathrm{di}$ ameter) to permit the circulation of water under the floor. The cylin-
} 
der stood in a water bath whose temperature could be maintained at a particular value $\left( \pm 0.5^{\circ} \mathrm{C}\right)$ by a Grant VFK Open Bath Circulator. The TF apparatus consisted of a $500-\mathrm{W}$ projection bulb housed within a fan-forced, air-cooled stainless steel box $(25 \mathrm{~cm} \times 16 \mathrm{~cm} \times$ $12 \mathrm{~cm}$ high) (cf. Helmstetter \& Bellgowan, 1993; J. M. Walker \& Dixon, 1983). A 2-mm aperture was located on the surface of the box directly above the bulb and between aluminium side rails $(3 \mathrm{~mm}$ high). A photocell, connected to a digital timer, was mounted $12 \mathrm{~cm}$ above the aperture. Lateral deflection of the rat's tail activated the photocell, terminating the light source and the digital timer. Latencies to TF were measured to the nearest millisecond. The heat source was adjusted to produce baseline TF latencies of approximately $3 \mathrm{sec}$ in naive rats. In the event that no TF occurred, the light was automatically terminated at $15 \mathrm{sec}$ to prevent tissue damage (Helmstetter \& Bellgowan, 1993). During TF testing, the rats were restrained by being wrapped individually with towelling material, with the entire tail exposed to allow for testing. The laboratory also contained wooden boxes ( $30 \mathrm{~cm}$ long $\times 28 \mathrm{~cm}$ wide $\times 30 \mathrm{~cm}$ high), which served as chambers where the rats were kept in isolation when brought to the laboratory from the adjacent colony room.

\section{Drug}

Naloxone $\mathrm{HCl}$ (E. I. duPont de Nemours \& Co., Wilmington, DE) was dissolved in $0.9 \% \mathrm{w} / \mathrm{v}$ saline solution and injected at a dose of $2.5 \mathrm{mg} / \mathrm{kg}$. Naloxone and physiological saline were injected subcutaneously (s.c.) into the dorsal area of the neck at a volume of $1.0 \mathrm{ml} / \mathrm{kg}$.

\section{Procedure}

Familiarization. The rats were handled on each of 3 successive days and were assigned to four weight-matched groups $(n=5)$. On Days $1-3$, they were given an injection of saline and placed into the wooden boxes for $20 \mathrm{~min}$. They were then exposed for $2 \mathrm{~min}$ to the floor of the hot-plate apparatus. The temperature of the water surrounding this floor was maintained at $23^{\circ} \mathrm{C}(\mathrm{Amb})$. The floor of the hot-plate apparatus was cleaned with $0.5 \%$ acetic acid solution after exposure of each rat. On each of these days, the rats were also familiarized to the TF restraining procedure for $12 \mathrm{~min}$. There was an interval of at least $1.5 \mathrm{~h}$ between exposure to the $23^{\circ} \mathrm{C}$ floor and restraint.

Conditioning. On each of Days 4 and 5, the subjects in Group NalHot/Nal-Hot and Group Sal-Hot/Sal-Hot were given naloxone or saline, respectively, and placed into the wooden boxes for $20 \mathrm{~min}$. They were then exposed for $60 \mathrm{sec}$ to the floor of the hot-plate apparatus. The water surrounding this floor was maintained at $52^{\circ} \mathrm{C}$. The rats in Group Sal-Amb/Sal-Hot were injected with saline, placed into the wooden boxes for $20 \mathrm{~min}$, and then exposed to the $23^{\circ} \mathrm{C}$ floor on Day 4, and to the $52^{\circ} \mathrm{C}$ floor on Day 5. On these days, the rats in Group Sal-Amb/Sal-Amb were injected with saline, placed into the wooden boxes, and then exposed to the $23^{\circ} \mathrm{C}$ floor. At least $1.5 \mathrm{~h}$ after exposure to the $23^{\circ} \mathrm{C}$ or the $52^{\circ} \mathrm{C}$ floor, the rats that had received saline were given a home-cage injection of naloxone in order to control for any nonspecific effects of the drug, whereas those that had received naloxone were given a control injection of saline in their home cages. The latencies to first PL in response to placement on the heated floor were recorded with a digital stopwatch to the nearest $1 / 100 \mathrm{sec}$. On both training days, the rats were again familiarized to the restraint procedure. Intervals of at least $1.5 \mathrm{~h}$ separated the three separate procedures of (1) injection, exposure to the wooden boxes, and placement on the floor of the hotplate apparatus, (2) injection in the home cages, and (3) restraint.

Test. On Day 6, the rats were injected with saline and placed into the boxes for $20 \mathrm{~min}$. They were then tested for latencies to flick their tails.

\section{Results and Discussion}

The results were analyzed with two sets of planned, orthogonal contrasts. The first of these was written to test for between-group differences in PL latencies on the final conditioning day to confirm previous observations of hypoalgesic effects in rats fested with the hot-plate assay. The second set of contrasts was written to test for between-group differences in TF latencies on the test day. With $\alpha=.05, d f=1,16$, the critical $F$ s were 4.49 (Hays, 1988).

\section{Conditioning}

Day 4. There were no significant differences in PL latencies between the rats that had received naloxone on their initial exposure to the heated floor and those that had received saline (Group Nal-Hot/Nal-Hot vs. Group SalHot/Sal-Hot, $M \mathrm{~s}=7.33$ and 7.67 , respectively, $F<1.00$; see Figure 1).

Day 5. There was evidence for hypoalgesia induced by preexposure to the heat stressor, because there were significant differences in PL latencies between the rats previously exposed to the heated floor and those exposed to it for the first time (Group Nal-Hot/Nal-Hot + Group Sal-Hot/Sal-Hot vs. Group Sal-Amb/Sal-Hot; $F=11.57$, $p=.0036$ ). Furthermore, there was evidence that naloxone potentiated acquisition of hypoalgesia, because there were significant differences in latencies to PL between the rats that had received naloxone-heat stressor pairings and those that had received separate exposures to the heat stressor and the drug. That is, the contrast that tested for differences between Group Nal-Hot/Nal-Hot and Group Sal-Hot/Sal-Hot was significant $(F=6.47, p=.0217)$.

\section{Test}

Day 6. There was evidence that preexposure to the heated floor induced hypoalgesia, because there were significant differences in TF latencies between the rats that were never exposed to the heated floor and those that had previously been exposed to the heated floor (see Figure 1). The contrast that tested for differences between Group Sal-Amb/Sal-Amb and the other three groups was significant $(F=7.28, p=.0158)$. There was also evidence that naloxone given in combination with exposure to the heated floor comes to potentiate acquisition of hypoalgesia, because there were significant differences between the rats that had received naloxone-heat stressor pairings and those that had received separate exposures to the drug and the heated floor (Group Nal-Hot/Nal-Hot vs. Group Sal-Hot/Sal-Hot + Group Sal-Amb/Sal-Hot; $F=4.71, p=.0454)$. There were no reliable differences between the two groups that had received separate exposures to naloxone and the heated floor $(F<1.00)$.

\section{EXPERIMENT 2}

The results of Experiment 1 have confirmed two previous findings. First, rats come to acquire hypoalgesic responses when tested for latencies to PL when they have been exposed to a heat stressor (Greeley \& Westbrook, 1990; Westbrook et al., 1991). Second, acquisition of these hypoalgesic responses is potentiated when exposure to the heat stressor is paired with the opioid antagonist nalox- 

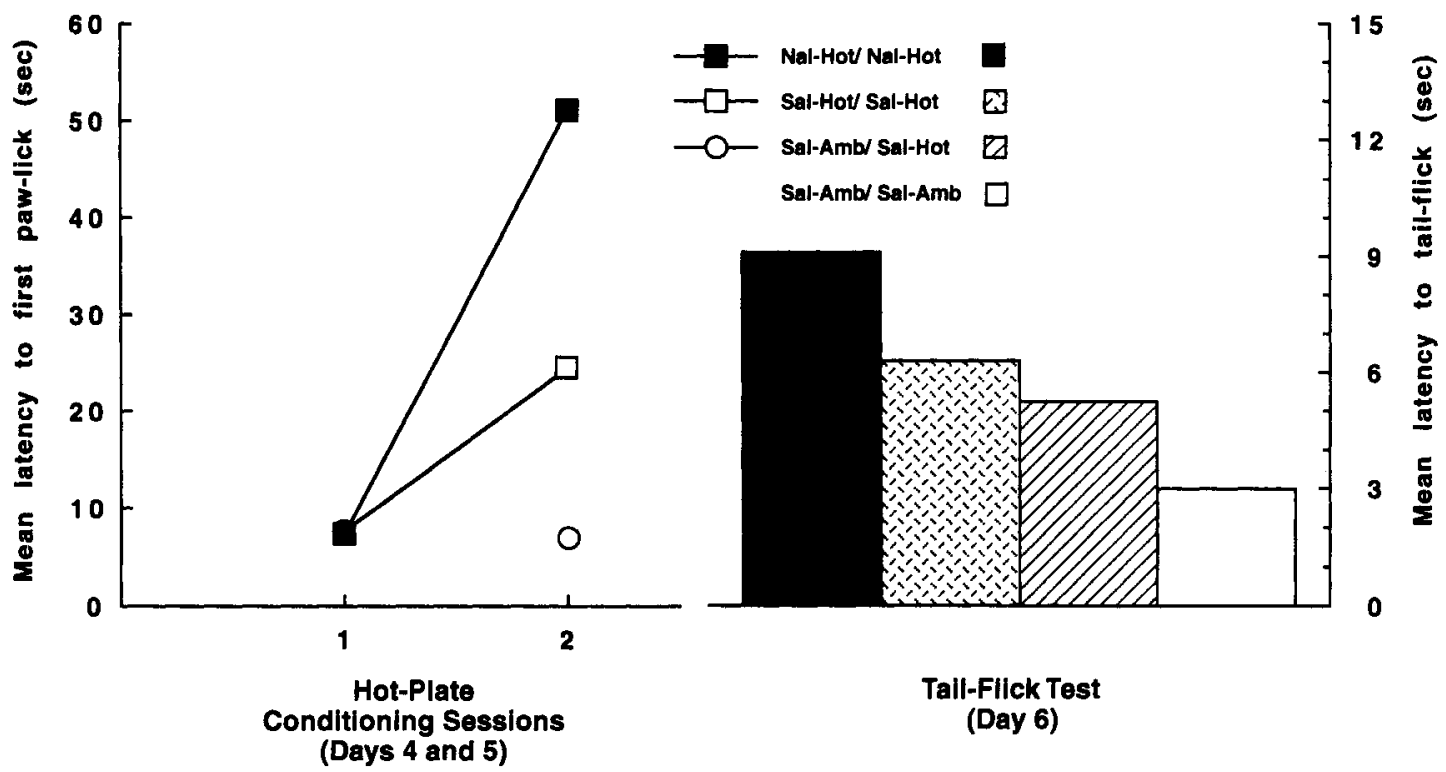

Figure 1. The left panel shows the mean latencies to first paw-lick for the rats in Group Nal-Hot/Nal-Hot and Group Sal-Hot/Sal-Hot across the two exposures to the heated floor, and for the rats in Group Sal-Amb/Sal-Hot on their single exposure to that floor. The right panel shows the mean latencies to tail-flick for each of these groups, as well as for Group Sal-Amb/Sal-Amb, on test.

one (Foo \& Westbrook, 1993; see also Foo, 1992, Foo \& Westbrook, 1991a, 1991b, 1991c, 1994, Poulos et al., 1990, Rochford \& Stewart, 1987a, Westbrook \& Greeley, 1990, and Westbrook et al., 1991). The results also revealed hypoalgesic responses in the rats tested for latencies to flick their tails in response to the application of radiant heat, providing further evidence for the generalizability of the hypoalgesic effects that have been detected previously. The aim of Experiment 2 was to determine the nature of the hypoalgesic responses observed with the TF test, using the criterion of naloxone reversibility to infer mediation by opioid mechanisms. Three main groups of subjects were used, with the rats in Group Nal-Hot receiving naloxone-heat stressor pairings, the rats in Group Sal-Hot receiving separate exposures to the drug and the heated floor, and the rats in Group SalAmb receiving separate exposures to naloxone and the nonheated floor, during conditioning. On test, half the subjects in each of these three groups were tested with naloxone (Nal), whereas the remaining subjects were tested with saline (Sal).

Method
Subjects
The subjects were 30 experimentally naive, male Wistar rats,
with an average weight of $224 \mathrm{~g}$ (range $160-260 \mathrm{~g}$ ). They were ob-
tained from the same source and were housed in conditions de-
scribed in Experiment 1 .
Apparatus and Drug
The apparatus and drug used were those described previously.
Procedure
Familiarization. The rats were familiarized to the apparatus, to
the injection procedure, and to restraint on each of the 3 days prior

to the start of the experiment proper. The procedures employed were those used in Experiment 1.

Conditioning. On Day 4, the subjects in Group Nal-Hot/Nal and Group Nal-Hot/Sal were injected with naloxone, whereas those in Group Sal-Hot/Sal and Group Sal-Hot/Nal were injected with saline, placed into the wooden boxes, and then exposed to the heated floor. The rats in Group Sal-Amb/Sal and Group Sal-Amb/Nal were given saline, placed into the wooden boxes, and then exposed to the $23^{\circ} \mathrm{C}$ floor. At least $1.5 \mathrm{~h}$ after exposure to the floor, the rats that had received saline were injected with naloxone, whereas those that had received naloxone were given saline. These injections took place in the colony room. The rats were also familiarized to the restraint procedure. Intervals of at least $1.5 \mathrm{~h}$ separated the three procedures of (1) injection and exposure to the wooden boxes and hot-plate apparatus floor, (2) injection in the home cages, and (3) restraint.

Test. On Day 5, the rats were brought to the laboratory and injected with naloxone (Group Nal-Hot/Nal, Group Sal-Hot/Nal, and Group Sal-Amb/Nal) or saline (Group Nal-Hot/Sal, Group Sal$\mathrm{Hot} / \mathrm{Sal}$, and Group Sal-Amb/Sal). They were placed into the boxes for $20 \mathrm{~min}$ and were then tested for latencies to TF. Five consecutive TF latencies were determined at 2 -min intervals. Repeated nociceptive assessment was employed because naloxone may have differential effects on hypoalgesic responses detected at an early (e.g., 1-2 min) versus later (e.g., 3-5 min) stage of testing (see Good \& Westbrook, 1995; Harris \& Westbrook, 1994). At least $1.5 \mathrm{~h}$ after exposure to the TF tests, the rats received an injection in their home cages. The rats tested with saline were injected with naloxone, whereas those tested with naloxone were injected with saline. On Day 6 , the rats were brought to the laboratory and injected with saline. They were placed into the wooden boxes for $20 \mathrm{~min}$ and then tested on the heated floor. This test was conducted to confirm that preexposure to the heated floor induced hypoalgesic effects that could be detected when the rats were tested with the hot-plate assay.

\section{Results and Discussion}

The analysis consisted of a set of planned, orthogonal contrasts written to test for between-group differences, a linear trend across the five TF tests, and groups $\times$ linear 
trend interaction effects. With $\alpha=.05, d f=1,24$, the critical $F$ s were 7.82 (group contrasts), 4.26 (linear trend), and 7.82 (group $\times$ linear trend interactions).

\section{Conditioning}

Day 4. There were no significant differences in PL latencies between the rats that had received naloxone and those that had received saline in advance of exposure to the heated floor $(F<1.00)$.

\section{Test}

Day 5. There was evidence that preexposure to the heated floor induced hypoalgesia, because there were significant differences in TF latencies between the rats preexposed to the heated floor and those preexposed to the nonheated one (Group Nal-Hot/Sal + Group NalHot/Nal + Group Sal-Hot/Sal + Group Sal-Hot/Nal vs. Group Sal-Amb/Sal + Group Sal-Amb/Nal; $F=41.983$, $p=.0000$; see Figure 2). There was also evidence that naloxone potentiated acquisition of hypoalgesia when given in combination with exposure to the heated floor. The contrast that tested for differences between the rats given naloxone-heat stressor pairing and those given separate exposures to the heat stressor and the drug during conditioning was significant (Group Nal-Hot/Sal + Group NalHot/Nal vs. Group Sal-Hot/Sal + Group Sal-Hot/Nal; $F=10.65, p=.0033$ ). Naloxone was able to reverse the hypoalgesic responses so induced, because there were re- liable differences between the rats preexposed to the heated floor and then tested for TF latencies with saline and those preexposed to the same floor and then tested for TF latencies with naloxone (Group Nal-Hot/Sal + Group Sal-Hot/Sal vs. Group Nal-Hot/Nal + Group Sal$\mathrm{Hot} / \mathrm{Nal} ; F=65.14, p=.0000$ ). There was no evidence that naloxone was differentially effective in reversing hypoalgesia in the rats given paired or separate exposures to naloxone and the heated floor, because the contrast that tested for an interaction effect was not significant $(F=4.63, p>.05)$. The reversal of hypoalgesia observed cannot be attributed to an unconditioned hyperalgesic effect of naloxone, because there were no reliable differences in TF latencies between the rats in Group Sal$\mathrm{Amb} / \mathrm{Sal}$ and those in Group Sal-Amb/Nal $(F<1.00)$. There was evidence for a general increase in TF latencies across repeated testing, because the contrast that tested for a linear trend was significant $(F=13.38, p=.0013)$. However, none of the group $\times$ linear trend interactions were significant, confirming naloxone's ability to reverse the long TF latencies across the five test trials.

Day 6. There was evidence that preexposure to the heated floor induced hypoalgesia, because there were significant differences between the rats preexposed to that floor and those preexposed to the nonheated one (Group Nal-Hot/Sal + Group Nal-Hot/Nal + Group SalHot/Sal + Group Sal-Hot/Nal vs. Group Sal-Amb/Sal + Group Sal-Amb/Nal; $F=12.31, p=.0018$; see Fig-

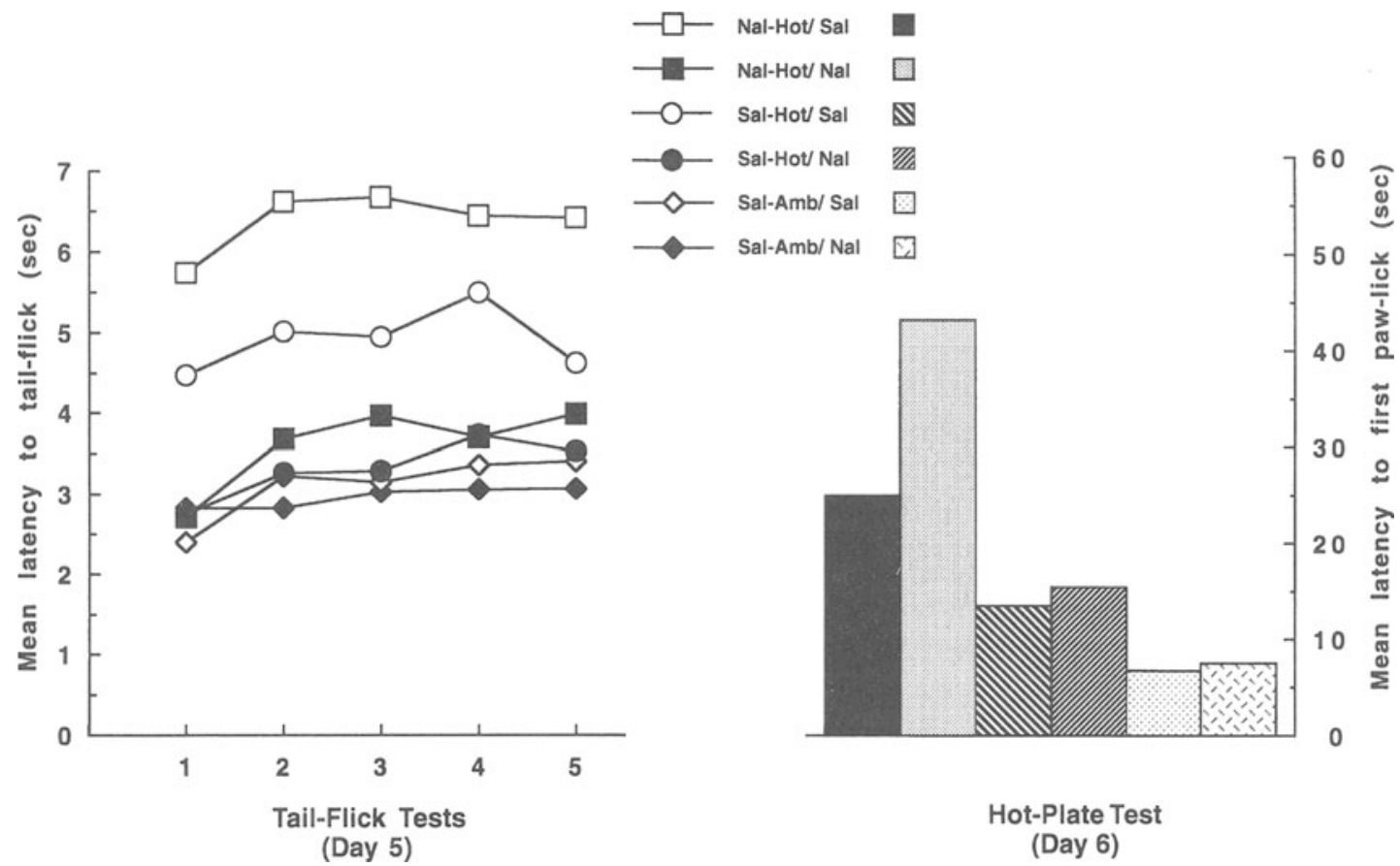

Figure 2. The mean latencies to tail-flick across the five consecutive test trials for the rats in each of the six groups in Experiment 2. The rats were given naloxone (Nal-) or saline (Sal-) before exposure to the heated (Hot) or ambient (Amb) floor on conditioning and were then tested for their latencies to tail-flick with either naloxone (Nal) or saline (Sal). The right panel shows the mean latencies to first paw-lick of the rats in each of the groups when they were tested with saline on the heated floor the following day. 
ure 2). There was also evidence that exposure to the heated floor with naloxone potentiated acquisition of hypoalgesia. The contrast that tested for differences between the rats given naloxone-heat stressor pairing and those given separate exposures to the drug and the heat stressor was significant (Group Nal-Hot/Sal + Group Nal-Hot/Nal vs. Group Sal-Hot/Sal + Group Sal-Hot/Nal; $F=12.11$, $p=.0019$ ).

\section{EXPERIMENT 3}

Experiment 2 showed that the hypoalgesic responses detected in rats tested for latencies to TF where they have received separate or combined exposures to naloxone and a heated floor were reversed by naloxone. Experiment 3 used a second criterion, that of cross-tolerance with morphine, to confirm that the hypoalgesic responses observed are mediated by opioid mechanisms. Six groups of rats were repeatedly injected in the colony room with either saline (Sal) or morphine (Mor), according to an injection regimen that has previously been shown to induce tolerance to the hypoalgesic effects of morphine (see Greeley \& Westbrook, 1990; Westbrook et al., 1991). The rats were then exposed to paired (Nal-Hot) or separate (Sal-Hot) exposures to naloxone and the heated floor or to separate exposures to naloxone and the nonheated floor (Sal-Amb). They were then tested with saline for latencies to TF where they had been exposed to the heated or nonheated floor.

\section{Method}

\section{Subjects}

The subjects were 30 experimentally naive, male Wistar rats, with an average weight of $225 \mathrm{~g}$ (range $140-270 \mathrm{~g}$ ). They were obtained from the same source and were housed in conditions described in Experiment 1

\section{Apparatus and Drugs \\ The apparatus and drugs used were those described previously. In addition, morphine $\mathrm{HCl}$ (Faulding) dissolved in $0.9 \%$ physiological saline was used.}

\section{Procedure}

Morphine/saline pretreatment (Days 1-12). After handling, the rats were allocated to six weight-matched groups $(n=5)$. The rats in three of these groups were injected with morphine (Group Mor/ Nal-Hot + Group Mor/Sal-Hot + Group Mor/Sal-Amb), whereas those in the other three groups received saline (Group Sal/Nal-Hot + Group Sal/Sal-Hot + Group Sal/Sal-Amb). These injections took place in the colony room. The drug dosage commenced at $5 \mathrm{mg} / \mathrm{kg}$ and was increased by $5 \mathrm{mg} / \mathrm{kg}$ every 3 days. By the end of the pretreatment period, the morphine-treated rats had received three injections of each of the four doses of morphine (i.e., $5,10,15$, and $20 \mathrm{mg} / \mathrm{kg}$ ), whereas the saline-treated rats received equivalent volumes of saline. The injection regimen was discontinued for 5 days to allow the rats to recover from any acute withdrawal.

Familiarization. On each of Days 15-17, the last 3 days of recovery from the morphine/saline injections, the rats were familiarized to the apparatus, the injection, and the restrain procedures in the manner described previously (see Method section of Experiment 1).

Conditioning. On Day 18 , the rats were brought to the laboratory, injected with naloxone ( $\mathrm{Nal})$ or saline (Sal), and placed into the wooden chambers for $20 \mathrm{~min}$. They were then exposed to the heated floor for $60 \mathrm{sec}$. The latencies to PL were recorded in the manner described previously.

Testing. On Day 19, the rats were brought to the laboratory, injected with saline, and placed into the wooden boxes for $20 \mathrm{~min}$. They were then tested for latencies to TF in the manner described in Experiment 2. On Day 20, the rats were brought to the laboratory, injected with saline, and placed into the wooden boxes for $20 \mathrm{~min}$. They were then tested for latencies to PL on the heated floor. This test was conducted to confirm that preexposure to the heated floor induced hypoalgesic effects that could be detected when the rats were tested with the hot-plate assay.

\section{Results and Discussion}

The analysis consisted of a set of planned, orthogonal contrasts written to test for between-group differences, a linear trend across the five TF tests, and groups $\times$ linear trend interaction effects. With $\alpha=.05, d f=1,24$, the critical $F$ s were 7.82 (group contrasts), 4.26 (linear trend), and 7.82 (group $\times$ linear trend).

\section{Conditioning}

Day 18. There were no significant differences in the latencies to first PL between the rats exposed to the heated floor on the conditioning day (Group Sal/Sal-Hot, $M=$ 6.124; Group Mor/Sal-Hot, $M=6.080$; Group Sal/NalHot, $M=6.098$; Group Mor $/$ Nal-Hot, $M=6.016$; all $F \mathrm{~s}<1.0)$.

\section{Test}

Day 19 (with saline on TF). There were significant differences in TF latencies between rats pretreated with saline and those pretreated with morphine (Group Sal/ Sal-Amb + Group Sal/Sal-Hot + Group Sal/Nal-Hot vs. Group Mor/Sal-Amb + Group Mor/Sal-Hot + Group Mor/ Nal-Hot; $F=89.46, p=.0000$; see Figure 3 ). An examination of the data reveals that the saline pretreated rats took longer to flick their tails than did the morphine pretreated ones. There was also evidence that preexposure to the heat stressor induced hypoalgesia, because there were significant differences in TF latencies between those preexposed to the heated floor and those preexposed to the nonheated one (Group Sal/Sal-Hot + Group Sal/NalHot + Group Mor/Sal-Hot + Group Mor/Nal-Hot vs. Group Sal/Sal-Amb + Group Mor/Sal-Amb; $F=19.26$, $p=.0002)$. The contrast that tested for an interaction between type of pretreatment (Sal vs. Mor) and temperature of floor (Hot vs. Amb) was significant $(F=53.26$, $p=.0000$ ), revealing that acquisition of hypoalgesia was differentially affected by pretreatment. As can be seen from Figure 3, the rats pretreated with saline acquired hypoalgesia, whereas those pretreated with morphine did not do so. There was also evidence that naloxone potentiated acquisition of hypoalgesia among the saline-pretreated rats, because there were reliable differences between Group Sal/Sal-Hot and Group Sal/NalHot $(F=12.68, p=.0016)$. However, a similar enhancement in acquisition of hypoalgesia was not evident among the morphine-pretreated rats that were exposed to the 


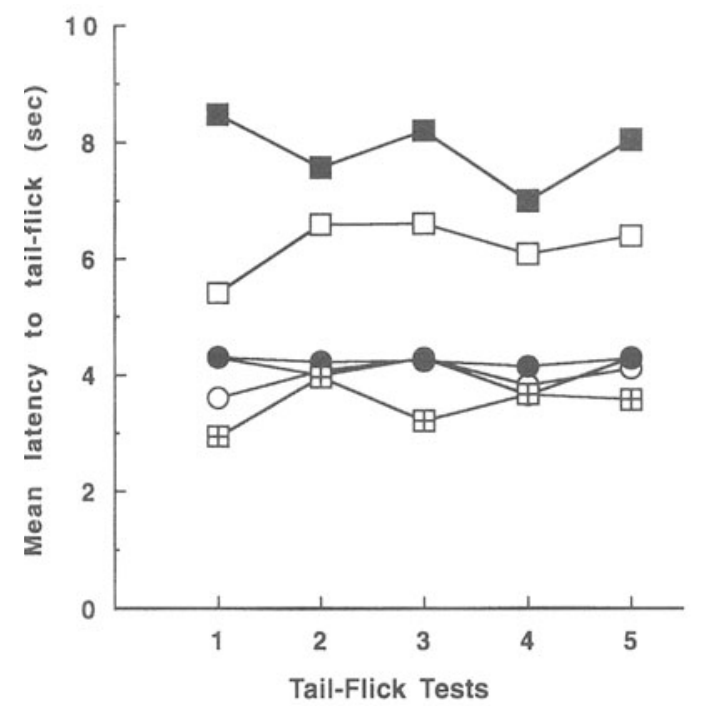

(Day 19)

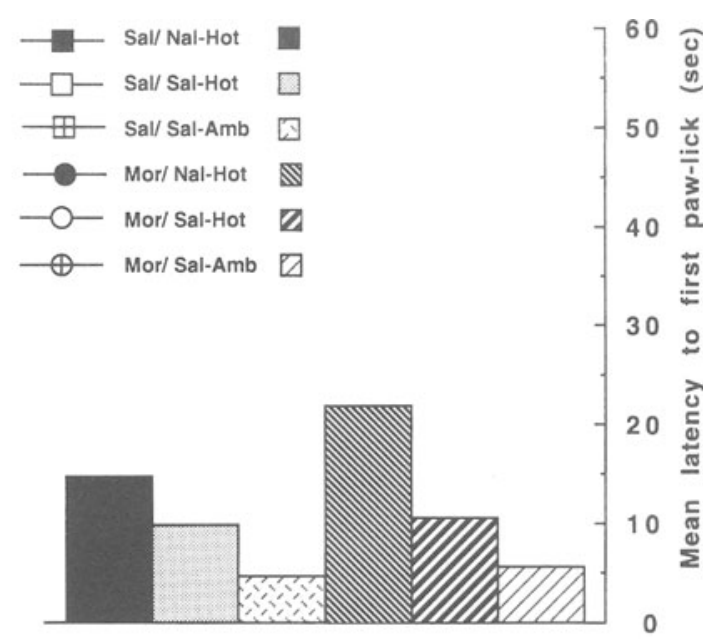

Hot-Plate Test (Day 20)

Figure 3. The left panel shows the mean latencies to tail-flick across the five consecutive test trials for the rats in each of the six groups in Experiment 3. The rats were pretreated with morphine (Mor) or saline (Sal) before conditioning with naloxone (Nal-) or saline (Sal-) on the heated (Hot) or nonheated/ambient (Amb) floor. They were then tested with saline for latencies to tail-flick. The right panel shows the mean latencies to first paw-lick of the rats in each of the groups when they were tested with saline on the heated floor the following day.

heated floor with naloxone (Group Mor/Sal-Hot vs. Group Mor/Nal-Hot; $F<1.0$ ). There was also no evidence for a linear change in TF latencies across test $(F<$ $1.00)$. Finally, none of the group $\times$ linear trend interactions were significant $(p>.05)$.

Day 20 (with saline on HP). The contrast that tested for differences between morphine- and saline-pretreated rats was significant (Group Mor/Sal-Amb + Group Mor/ Sal-Hot + Group Mor/Nal-Hot vs. Group Sal/Sal-Amb + Group Sal/Sal-Hot + Group Sal/Nal-Hot; $F=6.55, p=$ .0172 ; see Figure 3 ). An examination of the data shows that the morphine-pretreated rats took longer to lick their paws than did the saline-pretreated ones. There were also significant differences in PL latencies between the rats preexposed to the heated floor and those preexposed to the nonheated one, confirming that preexposure to the heated floor induced hypoalgesia (Group Sal/Sal-Hot + Group Sal/Nal-Hot + Group Mor/Sal-Hot + Group Mor/ Nal-Hot vs. Group Sal/Sal-Amb + Group Mor/Sal-Amb; $F=54.79, p=.0000$ ). The contrast that tested for an interaction between type of pretreatment (Sal vs. Mor) and temperature of floor (hot vs. amb) was not significant $(F=1.50, p>.05)$, providing evidence for comparable levels in acquisition of hypoalgesia among the salineand the morphine-pretreated rats. Furthermore, there was evidence that acquisition of hypoalgesia was potentiated by combining exposure to the heated floor with naloxone (Group Sal/Sal-Hot vs. Group Sal/Nal-Hot, $F=$ $5.93, p=.0227$; Group Mor/Sal-Hot vs. Group Mor/NalHot, $F=32.32, p=.0000$ ).

\section{EXPERIMENT 4}

Taken together, the results of Experiments 2 and 3 have provided evidence for opioid mechanisms in mediating hypoalgesic responses induced by separate or combined exposures to naloxone and a heat stressor. There is substantial evidence that opioid mechanisms of nociceptive inhibition can be modulated by the neuropeptide CCK. Specifically, there is evidence that CCK can come to antagonize the hypoalgesic effects of endogenous opioids. For example, CCK has been shown to attenuate (1) opioid hypoalgesic responses produced by external application of endogenous opioids (Itoh, Katsuura, \& Maeda, 1982), (2) unconditioned opioid hypoalgesic responses elicited by exposure to front-paw shock (Faris, Komisaruk, Watkins, \& Mayer, 1983), and (3) conditioned opioid hypoalgesic responses evoked by contextual cues associated with shock to the front paws (Faris et al., 1983). Such findings suggest that CCK might also serve to attenuate expression of the opioid-mediated hypoalgesic responses that have been detected in rats tested with the TF paradigm (see Experiments 2 and 3). Consequently, the aim of Experiment 4 was to examine the effects of cholecystokinin octapeptide (CCK-8) on expression of the long TF latencies observed in rats tested in a place where they had been exposed to a heat stressor. Three main groups of subjects were used, with the rats in Group NalHot receiving naloxone-heat stressor pairing, the rats in Group Sal-Hot receiving separate exposures to the drug and the heat stressor, and those in Group Sal-Amb receiv- 
ing separate exposures to naloxone and the nonheated floor during conditioning. On test, half the subjects in each of these three groups were tested with CCK-8 (CCK), whereas the remaining subjects were tested with saline (Sal).

\section{Method}

\section{Subjects}

The subjects were 30 experimentally naive, male Wistar rats, with an average weight of $262 \mathrm{~g}$ (range 210-320 g). They were obtained from the same source and were housed in conditions described in Experiment 1.

\section{Apparatus and Drugs \\ The apparatus and drugs used were those described in Experi- ment 1 . In addition, CCK-8 (Bristol-Myers Squibb Co., USA) dis- solved in $0.9 \% \mathrm{w} / \mathrm{v}$ saline was used. Naloxone was injected s.c. in the dorsal neck area at a dose of $2.5 \mathrm{mg} / \mathrm{kg}$. CCK-8 was injected i.p. at $3 \mu \mathrm{g} / \mathrm{kg}$. This dose was chosen because it has been shown to be effective in reversing conditioned opioid-mediated hypoalgesic re- sponses (see Faris et al., 1983).}

\section{Procedure}

Familiarization. After handling, the rats were allocated to six weight-matched groups ( $n=5$ ). They were then familiarized to the apparatus, to the injection procedures, and to restraint on each of the 4 days prior to the start of the experiment proper. An additional familiarization day was used in Experiment 4 to equate the number of s.c. and i.p. injections that the rats received during this period. The rats were familiarized with both routes of administration, receiving alternate s.c. and i.p. injections of saline across the 4 days. In all other respects, the procedures employed were similar to those described above (see Method section of Experiment 1).

Conditioning. On Day 5, the subjects in Group Nal-Hot/Sal and Group Nal-Hot/CCK were brought to the laboratory, injected with naloxone, and placed into the wooden boxes for $20 \mathrm{~min}$. They were then exposed for $60 \mathrm{sec}$ to the heated floor. The rats in Group SalHot/Sal and Group Sal-Hot/CCK were injected with saline, placed into the wooden boxes for $20 \mathrm{~min}$, and then exposed to the heated floor. The rats in Group Sal-Amb/Sal and Group Sal-Amb/CCK were given saline, placed into the wooden boxes, and then exposed to the $23^{\circ} \mathrm{C}$ floor for $60 \mathrm{sec}$. At least $1.5 \mathrm{~h}$ after exposure to the $23^{\circ} \mathrm{C}$ or the $52^{\circ} \mathrm{C}$ floor, the rats received an injection in their home cages. The rats that had received saline were injected with naloxone, whereas those that had received naloxone were given saline. The rats were also familiarized to the restraint procedure. Intervals of at least $1.5 \mathrm{~h}$ separated the three procedures of (1) injection and exposure to the wooden boxes and hot-plate apparatus floor, $(2)$ injection in the home cages, and (3) restraint.

Test. On Day 6, the rats were brought to the laboratory and injected with CCK-8 (Group Nal-Hot/CCK, Group Sal-Hot/CCK, and Group Sal-Amb/CCK) or saline (Group Nal-Hot/Sal, Group SalHot/Sal, and Group Sal-Amb/Sal). They were placed into the boxes for $20 \mathrm{~min}$ and were then tested for latencies to TF according to the procedure described previously (see Method section of Experiment 2). At least $1.5 \mathrm{~h}$ after exposure to the TF tests, the rats received an injection in their home cages. The rats tested with saline were injected with $\mathrm{CCK}$, whereas those tested with $\mathrm{CCK}$ were injected with saline. On Day 7 , the rats were brought to the laboratory and injected with saline. They were placed into the wooden boxes for $20 \mathrm{~min}$ and then tested on the heated floor. This test was conducted to confirm that preexposure to the heated floor induced hypoalgesic effects that could be detected when the rats were tested with the hot-plate assay.

\section{Results and Discussion}

The analysis consisted of a set of planned, orthogonal contrasts written to test for between-group differences, a linear trend across the five TF tests, and groups $\times$ linear trend interaction effects. With $\alpha=.05, d f=1,24$, the critical $F$ s were 7.82 (group contrasts), 4.26 (linear trend), and 7.82 (group $\times$ linear trend interactions).

\section{Conditioning}

Day 5. There were no significant differences in PL latencies between the rats that had received naloxone and those that had received saline in advance of exposure to the heated floor $(F<1.00)$.

\section{Test}

Day 6. There was evidence that preexposure to the heated floor induced hypoalgesia, because there were significant differences in TF latencies between the rats preexposed to the heated floor and those preexposed to the nonheated one (Group Nal-Hot/Sal + Group NalHot/CCK + Group Sal-Hot/Sal + Group Sal-Hot/CCK vs. Group Sal-Amb/Sal + Group Sal-Amb/CCK; $F=$ $17.25, p=.0004$; see Figure 4). However, the contrast that tested for differences between the rats given naloxoneheat stressor pairings and those given separate exposures to the heat stressor and the drug during conditioning was not significant (Group Nak-Hot/Sal + Group Nal-Hot/ CCK vs. Group Sal-Hot/Sal + Group Sal-Hot/CCK; $F=1.51, p>.05$ ). The hypoalgesic responses detected were reversed by CCK, because there were significant differences between the rats preexposed to the heated floor and then tested for TF latencies with saline and those preexposed to the same floor and then tested for TF latencies with CCK (Group Nal-Hot/Sal + Group Sal-Hot/Sal vs. Group Nal-Hot/CCK + Group Sal-Hot/CCK; $F=$ $80.26, p=.0000$ ). CCK was differentially effective in reversing hypoalgesia in the rats given paired or separated exposures to naloxone and the heated floor, because the contrast that tested for an interaction effect was significant $(F=9.85, p=.0045)$. As can be seen in Figure $4, \mathrm{CCK}$ was more effective in reversing hypoalgesia among the rats that had received naloxone-heat stressor pairing than among those that had received separate exposure to naloxone and the heated floor (Group Nal-Hot/ Sal - Group Nal-Hot/CCK vs. Group Sal-Hot/SalGroup Sal-Hot/CCK). The reversal of hypoalgesia is not due to an unconditioned hyperalgesic effect of CCK, because there were no reliable differences in TF latencies between the rats in Group Sal-Amb/Sal and those in Group Sal-Amb/CCK $(F<1.00)$. There was also no evidence for a general increase in TF latencies across repeated testing, because the contrast that tested for a linear trend was not significant $(F=1.48, p>.05)$. Furthermore, none of the group $\times$ linear trend interactions were significant, confirming CCK's ability to reverse the long TF latencies across the five test trials. 


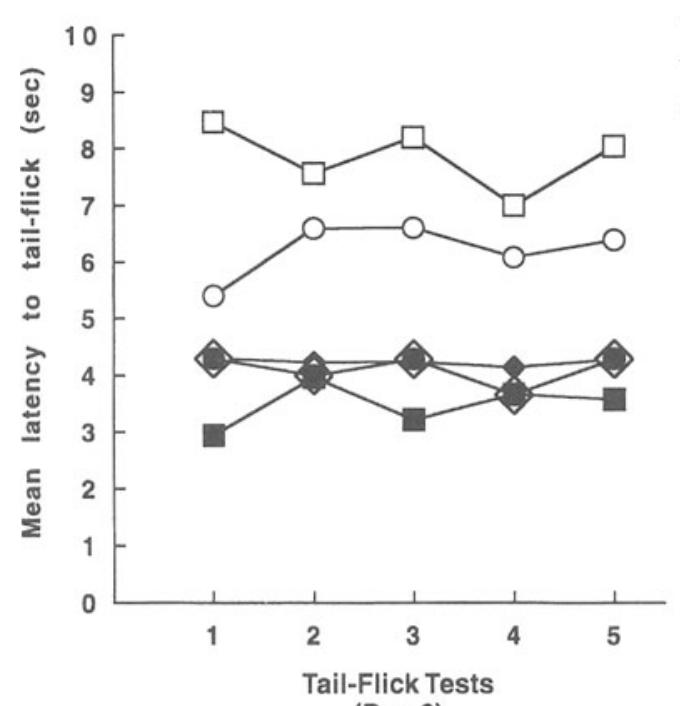

(Day 6)

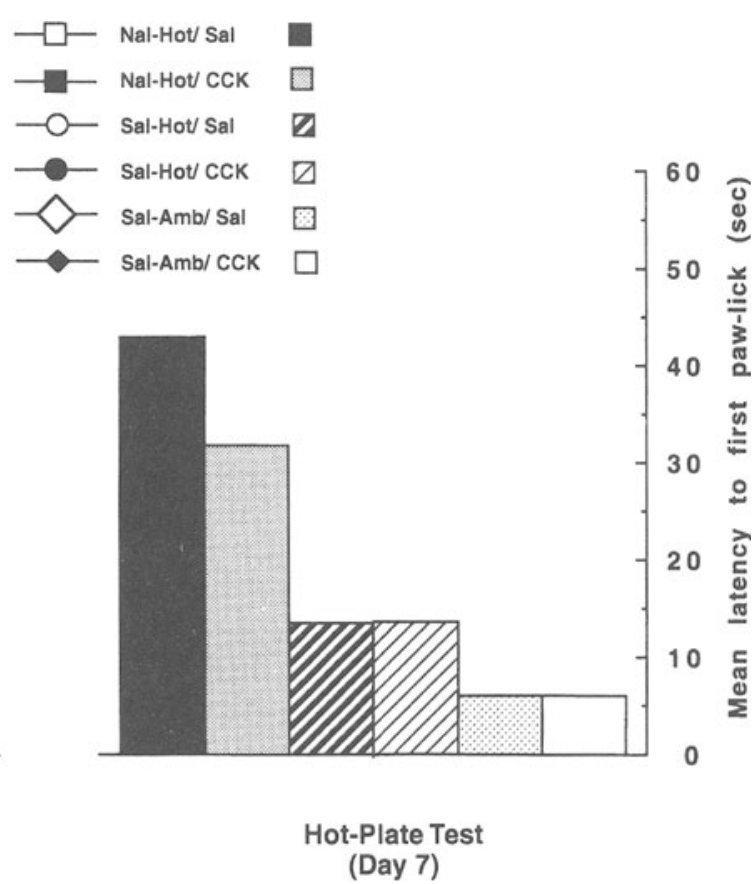

Figure 4. The mean latencies to tail-flick across the five consecutive tests for the rats in each of the six groups in Experiment 4. The rats were given naloxone (Nal-) or saline (Sal-) before exposure to the heated (Hot) or nonheated (Amb) floor on conditioning and were then tested for their latencies to tail-flick with either CCK-8 (CCK) or saline (Sal). The right panel shows the mean latencies to first paw-lick of the rats in each of the groups when tested with saline on the heated floor the next day.

Day 7. There was evidence that the rats preexposed to the heated floor were hypoalgesic when tested for latencies to PL, because there were significant differences between the rats preexposed to the heated floor and those preexposed to the nonheated floor (Group Nal-Hot/Sal + Group Nal-Hot/CCK + Group Sal-Hot/Sal + Group SalHot/CCK vs. Group Sal-Amb/Sal + Group Sal-Amb/ CCK; $F=13.32, p=.0013$; see Figure 4 ). There was also evidence that exposures to the heated floor with naloxone potentiated acquisition of hypoalgesia. The contrast that tested for differences between the rats given naloxone-heat stressor pairings and those given separate exposures to the drug and the heat stressor was significant (Group Nal-Hot/Sal + Group Nal-Hot/CCK vs. Group Sal-Hot/Sal + Group Sal-Hot/CCK; $F=14.97$, $p=.0007)$.

\section{EXPERIMENT 5}

Experiment 4 showed that expression of the opioidmediated hypoalgesic responses observed in the rats tested for latencies to TF where they have been exposed to a heat stressor was reversed by CCK-8. Experiment 5 used the nonspecific CCK antagonist proglumide to examine whether blockade of $\mathrm{CCK}$ receptors produces enhancement of the hypoalgesic responses. Previous investigations have shown that proglumide can serve (1) to enhance opioid hypoalgesic responses produced by mor- phine, DALA, or front-paw shock, without affecting basal nociceptive sensitivity/reactivity (Watkins et al., 1985; Watkins, Kinscheck, \& Mayer, 1984) and (2) to reverse the development of morphine hypoalgesic tolerance (Kellstein \& Mayer, 1991; Watkins et al., 1984). Experiment 5 used three main groups of subjects, with the rats in Group Nal-Hot receiving naloxone-heat stressor pairing, the rats in Group Sal-Hot receiving separate exposures to the drug and the heat stressor, and the rats in Group SalAmb receiving separate exposures to naloxone and the nonheated floor during conditioning. On test, half the subjects in each of these three groups were tested with proglumide (Prog), whereas the remaining subjects were tested with vehicle (Veh).

\section{Method}

\section{Subjects}

The subjects were 30 experimentally naive, male Wistar rats, with an average weight of $280 \mathrm{~g}$ (range $205-320 \mathrm{~g}$ ). They were obtained from the same source and were housed in conditions described in Experiment 1.

\section{Apparatus and Drugs}

The apparatus and drugs used were those described previously (see Method section of Experiment 1). In addition, proglumide (Sigma, USA) was used. Proglumide was prepared with DMSObuffer $(1.2 \%)$. All drugs were administered at a volume of $1.0 \mathrm{ml} / \mathrm{kg}$. Naloxone was injected s.c. in the dorsal neck area at a dose of $2.5 \mathrm{mg} / \mathrm{kg}$. Proglumide was injected i.p. at $0.02 \mathrm{mg} / \mathrm{kg}$. This dose 
was chosen on the basis that it has been shown to be effective in enhancing morphine-induced hypoalgesia (cf. Watkins et al., 1984).

\section{Procedure}

Familiarization. After handling, the rats were allocated to six weight-matched groups $(n=5)$. They were then familiarized to the apparatus, to the injection procedures, and to restraint on each of the 4 days prior to the start of the experiment proper. The procedures employed were the same as those described in Experiment 4.

Conditioning. On Day 5, the subjects in Group Nal-Hot/Sal and Group Nal-Hot/Prog were brought to the laboratory, injected with naloxone, and placed into the wooden boxes for $20 \mathrm{~min}$. They were then exposed for $60 \mathrm{sec}$ to the floor of the hot-plate apparatus. The rats in Group Sal-Hot/Sal and Group Sal-Hot/Prog were injected with saline, placed into the wooden boxes for $20 \mathrm{~min}$, and then exposed to the heated floor. The rats in Group Sal-Amb/Sal and Group Sal-Amb/Prog were given saline, placed into the wooden boxes, and then exposed to the $23^{\circ} \mathrm{C}$ floor for $60 \mathrm{sec}$. At least $1.5 \mathrm{~h}$ after exposure to the $23^{\circ} \mathrm{C}$ or the $52^{\circ} \mathrm{C}$ floor, the rats received an injection in their home cages in order to control for any nonspecific effects of naloxone. The rats that had received saline were injected with naloxone, whereas those that had received naloxone were given saline. The rats were also familiarized to the restraint procedure. Intervals of at least $1.5 \mathrm{~h}$ separated the three procedures of (1) injection and exposure to the wooden boxes and hot-plate apparatus floor, (2) injection in the home cages, and (3) restraint.

Test. On Day 6, the rats were brought to the laboratory and injected with proglumide (Group Nal-Hot/Prog, Group Sal-Hot/ Prog, and Group Sal-Amb/Prog) or vehicle (Group Nal-Hot/Veh, Group Sal-Hot/Veh, and Group Sal-Amb/Veh). They were placed into the boxes for $20 \mathrm{~min}$ and were then tested for latencies to TF according to the procedures described previously (see Method sec- tion of Experiment 2). At least $1.5 \mathrm{~h}$ after exposure to the TF tests, the rats received an injection in their home cages. The rats tested with vehicle were injected with proglumide, whereas those tested with proglumide were injected with vehicle. On Day 7 , the rats were brought to the laboratory and injected with saline. They were placed into the wooden boxes for $20 \mathrm{~min}$ and then tested on the heared floor. This test was conducted to confirm that preexposure to the heated floor induced hypoalgesic effects that could be detected when the rats were tested with the hot-plate assay.

\section{Results and Discussion}

The analysis consisted of a set of planned, orthogonal contrasts written to test for between-group differences, a linear trend across the five TF tests, and groups $\times$ linear trend interaction effects. With $\alpha=.05, d f=1,24$, the critical $F$ s were 7.82 (group contrasts), 4.26 (linear trend), and 7.82 (group $\times$ linear trend interactions).

\section{Conditioning}

Day 5. There were no significant differences in PL latencies between the rats that had received naloxone and those that had received saline in advance of exposure to the heated floor $(F<1.00)$.

\section{Test}

Day 6. There was evidence that preexposure to the heated floor induced hypoalgesia, because there were significant differences in TF latencies between the rats preexposed to the heated floor and those preexposed to

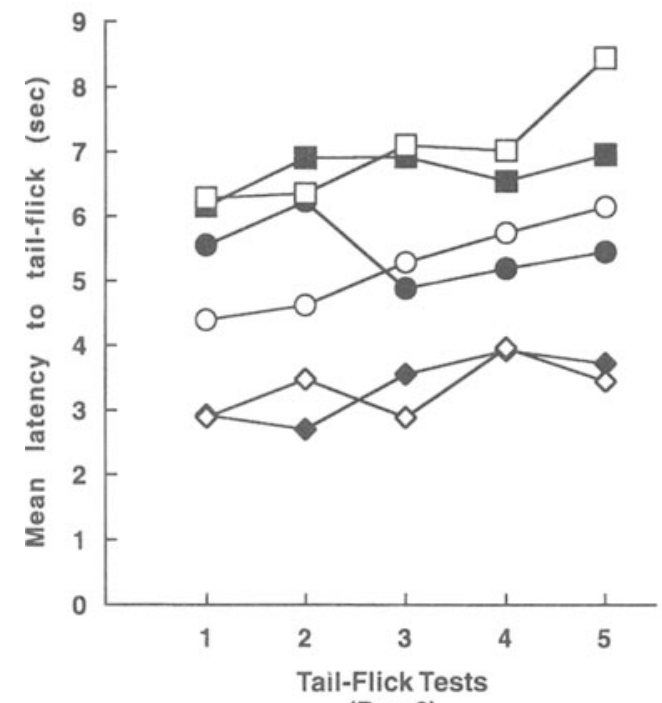

(Day 6)

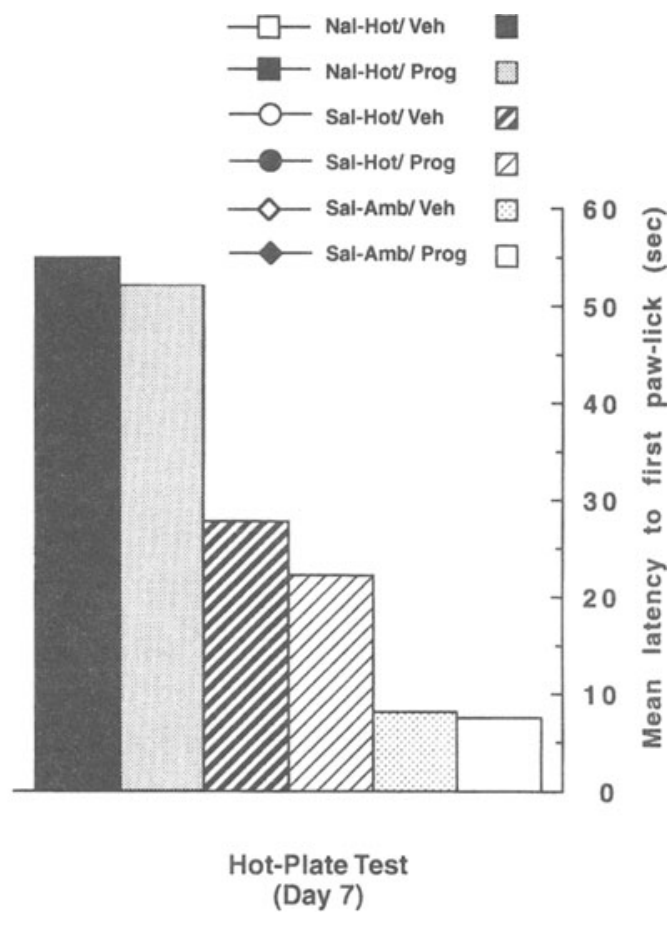

Figure 5. The mean latencies to tail-flick across the five consecutive tests for the rats in each of the six groups in Experiment 5. The rats were given naloxone ( $\mathrm{Nal}$-) or saline (Sal-) before exposure to the heated (Hot) or ambient (Amb) floor on conditioning and were then tested for their latencies to tail-flick with either proglumide (Prog) or vehicle (Veh). The right panel shows the mean paw-lick latencies of the rats in each of the groups when tested with saline on the heated floor the following day. 
the nonheated one (Group Nal-Hot/Veh + Group NalHot/Prog + Group Sal-Hot/Veh + Group Sal-Hot/Prog vs. Group Sal-Amb/Veh + Group Sal-Amb/Prog; $F=$ $62.03, p=.0000$; see Figure 5). There was evidence that naloxone potentiated acquisition of hypoalgesia when given in combination with exposure to the heated floor, because there were differences between the rats given naloxone-heat stressor pairing and those given separate exposures to the heat stressor and the drug (Group NalHot/Veh + Group Nal-Hot/Prog vs. Group Sal-Hot/ Veh + Group Sal-Hot/Prog; $F=15.46, p=.0006)$. The hypoalgesic responses detected were unaffected by proglumide, because there were no reliable differences between the rats preexposed to the heated floor and then tested for TF latencies with saline and those preexposed to the same floor and then tested for TF latencies with proglumide (Group Nal-Hot/Veh + Group Sal-Hot/Veh vs. Group Nal-Hot/Prog + Group Sal-Hot/Prog; $F<$ 1.0 ). There was also no evidence that proglumide affected basal nociceptive sensitivity/reactivity, because the contrast that tested for differences between Group Sal$\mathrm{Amb} / \mathrm{Sal}$ and Group Sal-Amb/Prog was not significant $(F<1.0)$. The contrast that tested for a linear trend across the five TF test trials was significant, indicating an increase in latencies $(F=10.73, p=.0032)$. However, none of the group $\times$ linear trend interactions were significant.

Day 7. There was evidence that the rats preexposed to the heated floor were hypoalgesic when tested for latencies to PL, because there were significant differences between the rats preexposed to the heated floor and those preexposed to the nonheated floor (Group Nal-Hot/Veh + Group Nal-Hot/Prog + Group Sal-Hot/Veh + Group SalHot/Prog vs. Group Sal-Amb/Veh + Group Sal-Amb/Prog; $F=35.73, p=.0000$; see Figure 5). There was also evidence that exposure to the heated floor with naloxone potentiated acquisition of hypoalgesia. The contrast that tested for differences between the rats given naloxone-heat stressor pairings and those given separate exposures to the drug and the heat stressor was significant (Group NalHot/Veh + Group Nal-Hot/Prog vs. Group Sal-Hot/Veh + Group Sal-Hot/Prog; $F=22.12, p=.0001$ ).

\section{EXPERIMENT 6}

The results from Experiments 1-5 have further confirmed that naloxone potentiates acquisition of hypoalgesic responses when its administration is paired with exposure to a heated floor of the hot-plate apparatus. The ability of naloxone either to maintain or to enhance acquisition of hypoalgesic responses is not specific to its interaction with a heated floor, but can be extended to a number of other types of stimuli. Specifically, such stimulation includes exposures to a novel environment (Foo \& Westbrook, 1991c; Rochford \& Dawes, 1992, 1993; Rochford, Dawes, \& Stewart, 1993), to a cold floor (Foo \& Westbrook, 1991c), to electric shock to the paws (Rochford \& Stewart, 1987a), and immersion of the rat's tail into heated water (Rochford \& Stewart, 1987a). This experiment examines whether naloxone interacts with the heat stimulus of the TF test used in the present investigation to induced hypoalgesic responses. Two main groups of rats were used, with rats in Group Nal-TF receiving naloxone in combination with testing for latencies to TF, and those in Group Sal-TF receiving unpaired exposures to the drug and the TF tests.

\section{Method}

\section{Subjects}

The subjects were 20 experimentally naive, male Wistar rats, with an average weight of $234 \mathrm{~g}$ (range 200-290 g). They were obtained from the same source and were housed in conditions described in Experiment 1.

\section{Apparatus and Drugs}

The apparatus and drugs used were those described previously (see Method section of Experiment 1).

\section{Procedure}

Familiarization. The rats were handled on each of 3 successive days and were assigned to weight-matched groups $(n=5)$. On Days 1-3, they were brought to the laboratory, given an injection of saline, and placed into the wooden boxes for $20 \mathrm{~min}$. They were then familiarized to the restraining procedure for $12 \mathrm{~min}$.

Test. On each of Days 4 and 5, the rats were taken to the laboratory and given an injection of naloxone (Nal) or saline (Sal). They were placed into the wooden boxes for $20 \mathrm{~min}$ and were then tested for latencies to $T F$ on three consecutive trials at 2 -min intervals. The rats were tested with a heat intensity that produced baseline latencies of approximately $3 \mathrm{sec}$. On Days 6 and 7, the rats in Groups Nal-TF $(3,6)$ and Sal-TF $(3,6)$ were tested with a lower heat intensity that produced baseline TF latencies of approximately $6 \mathrm{sec}$, whereas those in Groups Nal-TF $(3,3)$ and Sal-TF $(3,3)$ continued to be tested at the original heat intensity. A lower heat intensity was subsequently chosen for two of the groups in order to preclude any possibility that the test was insensitive in detecting hypoalgesic responses (i.e., floor effects). The TF latencies were recorded in the manner described previously. At least $1.5 \mathrm{~h}$ after exposure to the TF test, the rats received a control injection in their home cages. The rats that had received naloxone were injected with saline, whereas those that had received saline were given naloxone.

\section{Results and Discussion}

The analysis consisted of a set of planned, orthogonal contrasts written to test for between-group differences averaged across the 5 days of testing. With $\alpha=.05, d f=$ 1,16 , the critical $F$ was 4.49 . There was no evidence that naloxone interacts with the heat stimulus of the TF test to induce hypoalgesic responses, because there were no significant differences in TF latencies between the rats that had received naloxone paired with or separate from exposures to the TF tests [Group Nal-TF $(3,3)+$ Group Nal-TF $(3,6)$ vs. Group Sal-TF $(3,3)+$ Group Sal-TF $(3,6) ; F<1.0$; see Figure 6$]$. This absence of hypoalgesic responses was evident in the rats tested at both heat intensities, because the contrasts that tested for differences between Group Nal-TF $(3,3)$ versus Group Sal-TF $(3,3)$ and between Group Nal-TF $(3,6)$ versus Group Sal-TF $(3,6)$ were not significant $\left(F_{\mathrm{S}}<1.0\right)$. 


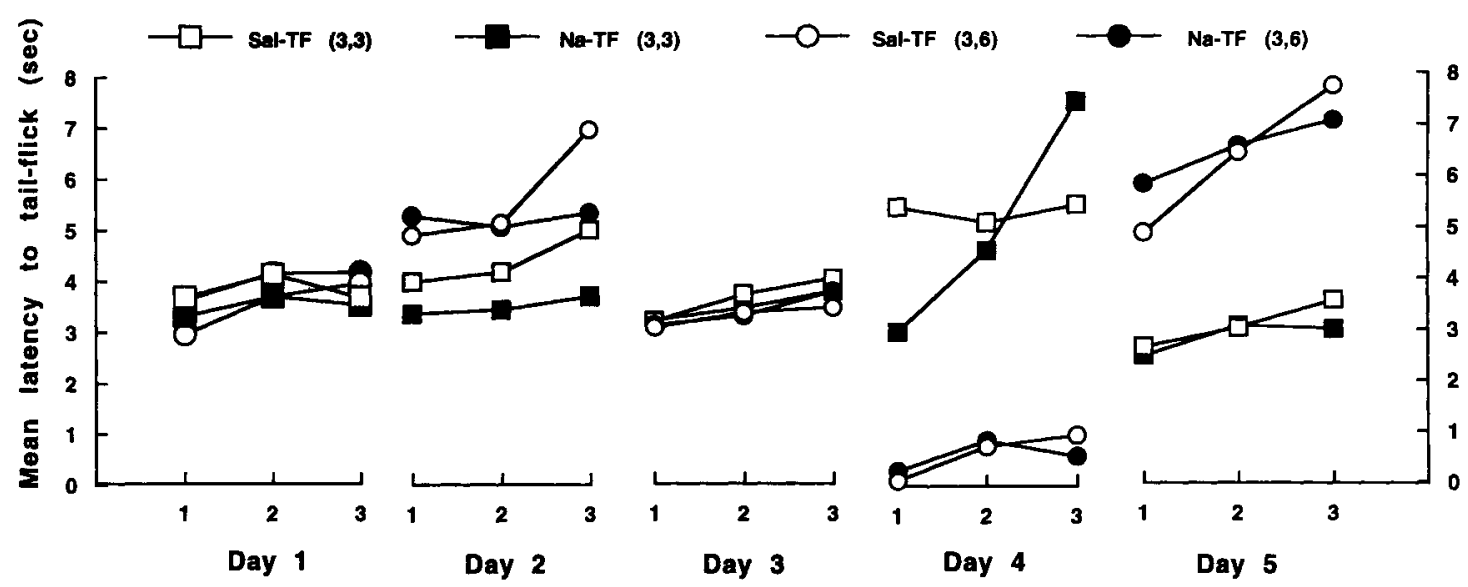

Figure 6. The mean latencies to tail-flick for the groups in Experiment 6 on each of the 5 days of testing. The rats were given naloxone (Na) or saline (Sal) before testing for latencies to flick their tails in response to a heat stimulus that produces baseline tail-flick latencies of approximately 3 or 6 sec.

\section{GENERAL DISCUSSION}

The present experiments have confirmed that rats given separate exposures to naloxone and to the heated floor of the hot-plate apparatus are hypoalgesic when subsequently tested on that floor, as evidenced by their long latencies to PL. They have also confirmed that naloxone given in combination with exposure(s) to the heated floor enhanced acquisition of the hypoalgesic responses, because the rats given such pairing(s) displayed longer latencies to PL. The present results have also shown that these effects are detected when the rats were tested for latencies to flick their tails in response to the application of radiant heat, providing further evidence for the generalizability of the hypoalgesic responses. In sum, these findings combine with those reported previously to document hypoalgesic responses resulting from preexposure to the heat stressor, and naloxone's ability to potentiate acquisition of these responses when given in combination with the heat stressor, across various pain assays (i.e., hot-plate, formalin, and TF).

The present results have also revealed that the hypoalgesic responses detected with the TF radiant heat test were reversed by naloxone (Experiment 2 ) and were not acquired by the rats with a history of exposures to morphine (Experiment 3 ). These findings thus suggest that the hypoalgesic responses are mediated by opioid mechanisms. In contrast, several investigations have confirmed that the hypoalgesic responses detected in rats tested with the hot-plate assay where they have been exposed to the heated floor were not reversed by naloxone and were not cross-tolerant with morphine, suggesting mediation by nonopioid mechanisms (e.g., Foo, 1992; Greeley \& Westbrook, 1990; Westbrook \& Greeley, 1990; Westbrook et al., 1991).

The distinctiveness of the responses observed with the TF and hot-plate tests is further evidenced by the differences in which the subjects in Experiment 3 responded to these tests. It may be recalled that this experiment employed six groups of rats, three of which were exposed to a morphine regimen previously shown to be effective in producing tolerance to the hypoalgesic effects of the drug, and the remaining three groups were given control injections of saline (cf. Westbrook et al., 1991). They were then exposed to a pairing of naloxone and the nonheated floor (Group Sal/Sal-Amb and Group Mor/Sal$A m b$ ), to naloxone and the heated floor separately (Group $\mathrm{Sal} / \mathrm{Sal}-\mathrm{Hot}$ and Group Mor/Sal-Hot), or to a pairing of naloxone and the heated floor (Group Sal/Nal-Hot and Group Mor/Nal-Hot). Across the next 2 days, the rats were tested for latencies to TF and to PL. As expected, the saline-pretreated rats exposed to the heated floor were hypoalgesic, as evidenced by their long latencies to TF or PL (see Figure 3; Group Sal/Sal-Hot and Group Sal/ Nal-Hot). The results of particular interest come from the two comparable groups of rats pretreated with morphine (Group Mor/Sal-Hot and Group Mor/Nal-Hot). Specifically, rats pretreated with morphine and exposed to the heated floor failed to show hypoalgesic responses when tested for their latencies to TF, but then showed hypoalgesic responses when tested for their latencies to PL. Of course, it could be argued that application of radiant heat to the rat's tail served as an aversive noxious stimulus that imbued its associated environment with the ability to provoke conditioned hypoalgesic responses observed on the heated floor. However, such an argument is unlikely for two reasons. First, rats in Group Mor/SalHot were hypoalgesic, relative to those in Group Mor/ Sal-Amb, when tested for their latencies to PL. Yet, these groups were equated in their history of treatment with morphine and with naloxone, and they were alike in responding to the TF tests but differed in terms of their exposure to the heated or nonheated floor, respectively, on the conditioning trial. Second, morphine-pretreated rats that had been exposed to the heated floor with naloxone (Group Mor/Nal-Hot), like those given separate exposure 
to naloxone and the heated floor (Group Mor/Sal-Hot), failed to show hypoalgesic responses when tested for their latencies to TF. However, on the subsequent hot-plate test, rats in Group Mor/Nal-Hot displayed longer latencies to PL than did those in Group Mor/Sal-Hot, confirming that naloxone potentiated acquisition of hypoalgesic response. Again, rats in these two groups had similar experiences with morphine and with naloxone, and they responded in the same manner on the TF tests, differing only in terms of whether they had received separate (Group Mor/Sal-Hot) or paired (Group Mor/Nal-Hot) exposures to naloxone and the heated floor during the conditioning trial.

Consequently, these results have revealed that the pain test used is critical in determining not only the opioid versus nonopioid nature of the hypoalgesic responses but also whether such responses are expressed. Although Experiment 3 did not confirm whether pretreatment with morphine had rendered rats tolerant to the hypoalgesic effects of the drug, I have been able to replicate the present findings to confirm that expression of conditioned hypoalgesia in morphine-tolerant rats depends on the pain test used (Foo, 1997).

The results of Experiment 4 are consistent with the finding that CCK-8 reversed expression of conditioned hypoalgesia provoked by a context associated with frontpaw shock (Faris et al., 1983). Specifically, the results showed that CCK-8 attenuated the opioid-mediated hypoalgesic responses detected in rats tested with the TF assay. However, there was no evidence that expression of these hypoalgesic responses was enhanced by proglumide (see Watkins et al., 1985). Perhaps, the present failure to detect any potentiation by proglumide may be related to the single dose that was employed. Alternatively, it is possible that the lack of specificity of proglumide for CCK$A$ and CCK-B receptor subtypes and the systemic administration of the drug may have contributed to this failure, since recent evidence suggests that anti-opioid activity of CCK is selective to the blockade of CCK-B receptors in the spinal cord (see Wiertelak, Maier, \& Watkins, 1992). Consequently, future investigations might employ injections of CCK-A and CCK-B at spinal level to determine the relative importance of CCK-A and CCK-B receptors in modulating the opioid-mediated hypoalgesic responses that have been observed.

The final experiment of this series investigated whether naloxone interacted with the heat stimulus used in the TF test to induce hypoalgesia. The results provided no evidence for hypoalgesic responses in rats given naloxone in combination with repeated exposures to the TF radiant heat test. In contrast, hypoalgesic effects have been reported in rats given naloxone in combination with exposures to the TF immersion test. In a demonstration of these effects, Rochford and Stewart (1987a, Experiment 3) employed four groups of rats that differed according to whether they received paired or unpaired exposures to naloxone and immersion of the rat's tail into a $45^{\circ} \mathrm{C}$ or a $50^{\circ} \mathrm{C}$ water bath. These investigators found that rats given naloxone in combination with the TF immersion test were hypoalgesic, relative to those given the drug separately from the tests. The discrepancy between these findings and those of Experiment 6 could be explained by procedural differences in the studies. In both investigations, the rats were familiarized with the restraining procedure required for TF testing before the start of the experiment proper. However, in the present study, the rats were also familiarized with the injection procedure, because naloxone has been shown to interact with the injection procedure to elicit hypoalgesic responses (see Foo \& Westbrook, 1991b). Since rats in the present study were familiarized with both the restraint and the injection procedures before receiving pairings of naloxone and the TF tests, the failure to detect hypoalgesia could be interpreted to mean that the heat stimulus itself was not sufficiently aversive to interact with naloxone to produce hypoalgesia. When combined with reports that naloxone interacts with the heat stimulus of the hot-plate test to either maintain or enhance acquisition of hypoalgesia, the present findings suggest that the TF radiant heat test might be a better pain assay to use in studies investigating stress-induced hypoalgesias. Furthermore, the present findings also suggest the use of multiple pain assays to assess nociceptive sensitivity/reactivity in morphine-experienced rats, since expression of conditioned hypoalgesic responses in such rats differ according to the test employed.

\section{REFERENCES}

Bianchi, M., \& Panerai, A. E. (1993). Naloxone-induced analgesia: Involvement of $\kappa$-opiate receptors. Pharmacology, Biochemistry \& Behavior, 46, 145-148.

Cox, J., \& Westbrook, R. F. (1994). The NMDA receptor antagonist MK-801 blocks acquisition and extinction of conditioned hypoalgesic responses in the rat. Quarterly Journal of Experimental Psychology, 47B, 187-210.

D'Amour, F. E., \& Smiтh, D. L. (1941). A method for determining loss of pain sensation. Journal of Pharmacology \& Experimental Therapeutics, 72, 74-79.

Faris, P. L., Komisaruk, B. R., Watkins, L. R., \& Mayer, D. J. (1983). Evidence for the neuropeptide cholecystokinin as an antagonist of opiate analgesia. Science, 219, 310-312.

Foo, H. (1992). The hypoalgesia conditioned to a heat stressor with naloxone is nonopioid: Implications for the hypoalgesias conditioned by shock. Psychobiology, 20, 51-64.

Foo, H. (1997). Expression of conditioned hypoalgesia in morphinetolerant rats depends on the pain test used. Manuscript submitted for publication.

Foo, H., \& WESTBROOK, R. F. (1991a). Effects of hypophysectomy and adrenalectomy on naloxone-induced hypoalgesia. Psychopharmacology, 103, 177-182.

FoO, H., \& WESTBROOK, R. F. (199 lb). Naloxone-induced hypoalgesia: Effects of heat, cold, and novelty. Quarterly Journal of Experimental Psychology, 43B, 137-156.

Foo, H., \& WestbroOK, R. F. (1991c). Naloxone-induced hypoalgesia: Effects of noradrenergic antagonists and agonist. Pharmacology, Biochemistry \& Behavior, 39, 795-797.

Foo, H., \& Westbrook, R. F. (1992). Exposure to a heat stressor induces an opioid conditioned hypoalgesia in rats tested for nociception to formalin. Society for Neuroscience Abstracts, 18, 1026. 
Foo, H., \& WESTBROOK, R. F. (1993). Naloxone-induced hypoalgesia: Evidence from the formalin test. Pharmacology, Biochemistry \& Behavior, 45, 501-505.

Foo, H., \& WESTBROOK, R. F. (1994). The form of the conditioned hypoalgesic response resulting from preexposure to a heat stressor depends on the pain test used. Psychobiology, 22, 173-179.

GALLAGHER, M. (1985). Re-viewing modulation of learning and memory. In N. H. Weinberger, J. L. McGaugh, \& G. Lynch (Eds.), Memory systems of the brain (pp. 311-334). New York: Guilford.

GoOD, A. J., \& WESTBROOK, R. F. (1995). Effects of microinjection of morphine into the amygdala on the acquisition and expression of conditioned fear and hypoalgesia in rats. Behavioral Neuroscience, 109 631-641.

Greeley, J. D., Le, A. D., Poulos, C. X., \& Cappell, H. (1988). "Paradoxical" analgesia induced by naloxone and naltrexone. Psychopharmacology, 100, 31-35.

GREELEY, J. D., \& WeStBROOK, R. F. (1990). Some effects of exposure to a heat stressor upon the rat's subsequent reactions to that stressor Quarterly Journal of Experimental Psychology, 42B, 241-265.

Grossman, G. L., Basbaum, A. L., \& Fieldos, H. (1982). Afferent and efferent connections of the rat tail flick reflex (a model used to analyze pain control mechanisms). Journal of Comparative Neurology, 206, 9-16.

HaRRIS, J. A., \& WESTBROOK, R. F. (1994). Effects of midazolam and naloxone in rats tested for sensitivity/reactivity to formalin pain in a familiar, novel or aversively conditioned environment. Psychopharmacology, 115, 65-72.

Hays, W. L. (1988). Statistics (4th ed.). New York: Holt, Rinehart \& Winston.

Helmstetter, F. J., \& Bellgowan, P. S. (1993). Lesions of the amygdala block conditional hypoalgesia on the tail-flick test. Brain Research, 612, 253-257.

Irwin, S., Houde, R. W., Bennett, D. R., Hendershot, L. C., \& SeevERS, M. H. (1951). The effects of morphine, methadone and mereperidine on some reflex responses of spinal animals to nociceptive stimulation. Journal of Pharmacology \& Experimental Therapeutics, 101, 132-143.

Itoh, S., Katsunra, G., \& MaEda, Y. (1982). Caerulein and cholecystokinin suppress b-endorphin-induced analgesia in the rat. European Journal of Pharmacology, 80, 421-425.

IZQUIERDO, I. (1979). Effects of naloxone and morphine on various forms of memory in the rat: Possible role of endogenous opiate mechanisms in memory consolidation. Psychopharmacology, 66, 199-203.

Kavaliers, M., \& InNES, D. G. L. (1993). Sex differences in naloxoneand Tyr-MIF-1-induced hypoalgesia. Peptides, 14, 1001-1004

KELLSTEIN, D. E., \& MAYER, D. J. (1991). Spinal co-administration of cholecystokinin antagonists with morphine prevents the development of opioid tolerance. Pain, 47, 221-229.

Litchman, A. H., \& Fanselow, M. S. (1990). Cats produce analgesia in rats on the tail-flick test: Naltrexone sensitivity is determined by nociceptive tests stimulus. Brain Research, 533, 91-94.
Poulos, C. X., KNoke, D. M., Le, A. D., \& Cappell, H. D. (1990). Naloxone-induced analgesia and morphine supersensitivity effects are contingent upon prior exposure to analgesic testing. Psychopharmacology, 100, 31-35.

RochFoRd, J., \& Dawes, P. (1992). Clonidine and yohimbine modulate the effects of naloxone on novelty-induced hypoalgesia. Psychopharmacology, 107, 575-580.

ROCHFORD, J., \& DAWES, P. (1993). Effect of naloxone on the habituation of novelty-induced hypoalgesia: The collateral inhibition hypothesis revisited. Pharmacology, Biochemistry \& Behavior, 46, 117-123.

ROCHFORD, J., DAWES, P., \& STEWART, J. (1993). Naloxone potentiation of novelty-induced hypoalgesia: Characterization of the a-noradrenergic receptor subtype. Pharmacology, Biochemistry \& Behavior, 44, 381-386.

RochFord, J., \& STEWART, J. (1987a). Activation and expression of endogenous pain control mechanisms in rats given repeated nociceptive tests under the influence of naloxone. Behavioral Neuroscience, 101, 87-103.

ROCHFORD, J., \& STEWART, J. (1987b). Morphine attenuation of conditioned autoanalgesia: Implications for theories of situation-specific tolerance to morphine analgesia. Behavioral Neuroscience, 101, 690-700.

Ross, R. T., \& RANDICH, A. (1985). Associative aspects of conditioned analgesia evoked by a discrete CS. Animal Learning \& Behavior, 13, 419-431.

WALKER, J. M., \& DiXoN, W. C. (1983). A solid state device for measuring sensitivity to thermal pain. Physiology \& Behavior, 30, 181-183.

Walker, M. K., Le, A. D., Poulos, C. X., \& Cappell, H. (1991). Role of central versus peripheral opioid receptors in analgesia induced by repeated administration of opioid antagonists. Psychopharmacology, 104, 164-166.

Watkins, L. R., Kinscheck, I. B., Kaufman, E. F. S., Miller, J., FRENK, H., \& MAYER, D. J. (1985). Cholecystokinin antagonists selectively potentiate analgesia induced by endogenous opiates. Brain Research, 327, 181-190.

Watkins, L. R., Kinscheck, I, B., \& Mayer, D. J. (1984). Potentiation of opiate analgesia and apparent reversal of morphine tolerance by proglumide. Science, 224, 395-396.

WESTBROOK, R. F., \& GREELEY, J. D. (1990). Some effects of the opioid antagonist, naloxone, upon the rat's reactions to a heat stressor. Quarterly Journal of Experimental Psychology, 42B, 1-40.

Westbrook, R. F., Greeley, J. D., Nabke, C. P., Swinbourne, A. L., \& HARVEY, A. (1991). Effects of morphine and naloxone upon the reactions of rats to a heat stressor. Quarterly Journal of Experimental Psychology, 43B, 323-346.

Wiertelak, E. P., Maier, S. F., \& Watkins, L. R. (1992). Cholecystokinin antianalgesia: Safety cues abolish morphine analgesia. Science, 256, 830-833.

(Manuscript received March 11, 1997; revision accepted for publication September 17, 1997.) 